\title{
Extended virtual in-situ calibration method in building systems using Bayesian inference
}

\author{
Sungmin Yoon, Yuebin $\mathrm{Yu}^{*}$ \\ Durham School of Architectural Engineering and Construction, University of Nebraska-Lincoln, \\ United States
}

Keywords: Building systems, Sensor calibration, Virtual in-situ calibration, Bayesian inference, Markov Chain Monte Carlo, Absorption Refrigeration

\begin{abstract}
:
Measurements from sensors and knowledge of key parameters are of great importance in the operation of modern building systems. Accurate and reliable information as these serves as the base for ensuring the desired performance of control algorithms, fault detection and diagnostics rules, analytical optimization strategies. They are also crucial for developing trust-worthy building models. However, unlike mass produced industrial devices, building systems are generally one of a kind and sparsely instrumented. Despite the indispensable need, dense deployment of sensors or a periodic manual calibration for ensuring the quality of thousands variables in building systems is not practical. To address the challenge, we extend our virtual in-situ calibration method by marrying it with Bayesian inference, which has a better capability in handling uncertainties. Strategies, including local, global, and combined calibration, are evaluated in a case with various sensor errors and uncertain parameters. The detailed procedure and results are presented.
\end{abstract}

\section{Introduction}

Energy used for heating, ventilation, air conditioning, and refrigeration systems (HVAC\&R) is a large part of the total energy consumed in buildings to provide a proper indoor environment for occupants [1,2]. Issues, including problematic or inferior control sequences and set points, equipment performance degradation and various faults occurring in HVAC\&R and building energy systems further increase energy use or can lead to undesired indoor environmental quality. To address the challenges in the building sector, a comprehensive solution package, including continuous fine-tuning of building automation systems, automated analytical optimization, and automated fault detection, diagnostics and repair, is needed. Research into these challenges has been conducted in order to help mitigate increasing energy use in buildings [3-8]. Most of the proposed approaches will be effective only if the data obtained from sensors are reliable and accurate [3]. In the field of smart buildings, the role of sensors is significant for ensuring and enhancing building performance.

Physical sensors that measure variables in terms of the building or system conditions are vulnerable to the working conditions and tend to have errors related to these vulnerabilities. The sensor errors can be categorized into two major types [9]: (1) systematic errors (bias or offset) and (2) random errors (noise). Systematic errors are indicated by the discrepancy between the mean of measurements and measurands (their true value). They may occur because of the sensor's physical condition, measured phenomena, working environments, or other factors. For instance, Yu. et al. [10] found that systematic errors of commonly preinstalled supply-air temperature sensors in compact rooftop air conditioners could be have errors up to $19.2^{\circ} \mathrm{C}$ due to their compact size, poor air distribution, and intensive thermal radiation of a gas heating chamber. Random errors are indicated by the difference between the measurements and their mean. They are caused by external problems that affect the sensor readings or hardware noise. Random errors can be detected by a probability distribution, such as Gaussian, in some cases. A random error with a large standard deviation indicates 
measurement inaccuracies. Regardless of how advanced the building automation and control algorithms are, these rudimentary errors from sensors adversely affect analysis and thereby lead to inferior building system performance.

Periodic calibration is needed to improve the reliability of measurements from working sensors. In a conventional calibration [11-15], working sensors can be calibrated by reducing the difference between the values obtained from working sensors or using benchmarks from standards and reference sensors in given conditions [14,15]. Usually, such a calibration requires a process of removing and reinstalling the sensor to its working environment. Considering the features of building sensor network, the conventional method has practical problems [16]: (1) time and monetary cost for reinstalling sensors; (2) disruption to a normal operation; (3) difficulty in accessing various sensors in pipelines, hidden spaces, etc.; and (4) different working environments with the performed conditions for a calibration. Recently, to overcome these limitations in building systems, Yu and $\mathrm{Li}$ [16] proposed a virtual in-situ calibration method. This method can determine the benchmarks that are either statistically established or mathematically modeled, without removing the embedded working sensor in a system or adding extra reference sensors as in a conventional calibration. More specifically, the statistical-based method determines a benchmark value by calculating a mean of measurements. It requires a redundancy of sensors for measuring the same phenomenon. This is useful for detecting random errors from individual working sensors when sensor redundancy exists. On the other hand, the modeling-based method can reduce both systematic and random errors without sensor redundancy. This method uses mathematical equations of a system model and known relative variables in the model to estimate the benchmark values of working sensors. The calibration function is determined by casting the problem as an optimization problem, i.e., minimizing the difference between the sensor's measurements and the benchmarks.

With respect to an operational building system, the relative or dependent variables used in the benchmark model for a specific working sensor may be defined by (1) measurements from other working sensors in a system and (2) parameters in the system model. There are uncertainties from both of the sources. Even though all relative variables of the benchmark are defined by measurements from other sensors, which might have higher reliability than the target working sensor itself, we cannot assure that the measured values are always accurate. The accuracy is subject to influences from the physical environment when the measurements are taken. As well, parameters may naturally contain uncertainties not accounted for when they were determined, e.g. a heat transfer coefficient, thermal capacitance, etc. Because of these potential inaccuracies, it is more beneficial to consider all critical working sensors of a system and relevant unknown parameters of the system in order to model a virtual in-situ, modeling-based calibration process.

When a large number of unknowns is included in a mathematical calibration, the problem becomes under-determined. It means that the total number of unknown variables composed of relative variables and unknown parameters can be inherently greater than that of equations provided from a system model. It is impossible to calculate the accurate benchmarks for every unknown variable in an under-determined calibration problem. Several modeling-based methods have been proposed in different research fields to convert these to determined problems, such as an on-line calibration [17], a collaborative calibration [9], a blind calibration [18], and a self-calibration [19]. In chemistry, various calibration methods [20-23] use more than one reference sensor for benchmarks, which can be considered as a known calibration environment. Literature in this regard mainly come from fields where sensor redundancy, high quality sensors, or known relationships between sensors does exist. Unfortunately, it is considerably difficult to adopt any of these approaches in a building sensor 
network because a building system is not mass produced or well instrumented. There is also a limited number and quality of sensors for each phenomenon. As well, many sensors are needed for various building phenomena at different locations and levels (temperatures, mass flow rates, pressures, etc.).

We argue that, compared to other fields, building systems are complicated and stochastic with multiple unknown parameters and uncertainties, especially when occupants, devices, and ambient environment all interact. This paper proposes to extend virtual in-situ calibration methods by adding a probabilistic formulation. This approach considers the characteristic of building sensor networks, typical errors of building sensors, and limitations of existing calibration methods when applying to an entire building system. We first briefly present the algorithm of extended virtual in-situ calibration. Then, we describe the Monte Carlo Markov Chain (MCMC) approach to solving the problem. To verify the suggested method, the algorithm is applied to a $\mathrm{LiBr}-\mathrm{H} 2 \mathrm{O}$ absorption refrigeration system in a virtual environment with pre-defined true values. 


\section{Extended virtual in-situ calibration formulating using Bayesian inference}

\subsection{Benchmark and correction function in virtual in-situ calibration}

Conventional and virtual in-situ calibration is driven by an objective function. It can be expressed as shown in Eq. (1). In this objective function, the measurements from working sensors are corrected by minimizing the distance between the measured values and the corresponding benchmark values that are defined by standards or reference sensors as in Eq. (2).

$$
\begin{aligned}
& \min _{M} D(M)=\left|Y_{b}-M\right| \\
& Y_{b}=S \quad \text { or } \quad Y_{b}=M_{R}
\end{aligned}
$$

where, $D$ is the distance function regarding the measurement error, $M$ is the measurement from the working sensor, $Y_{b}$ is the corresponding benchmark for the working sensor, $S$ is the known standard value for the working sensor, and $M_{R}$ is the measurement from the corresponding reference sensor.

In EVIC (extended virtual in-situ calibration), the benchmarks are mathematically estimated by equations and relative variables $\left(v_{r}\right)$ in a system model, as in Eq. (3), without the additional reference sensors or standards.

$$
Y_{b}=f\left(v_{1}, v_{2}, v_{3}, \ldots, v_{r}\right)
$$

where, $v$ is the relative variable of the measurement $M, r$ is the counter for the relative variables, and $f$ is the system model.

Since the relative variables may be defined by measurements from other working sensors or unknown parameters of the model, the benchmarks can be rewritten as Eq. (4). It is deemed that the calculated values can approximate the benchmark values.

$$
Y_{b}=f\left(M_{v 1}, M_{v 2}, \ldots, M_{v r}, x_{u 1}, x_{u 2}, \ldots, x_{u q}\right)
$$

where, $M_{v r}$ is the measured values of the $r$ th relative variables, $x_{u}$ is the unknown parameter in the system model, and $q$ is the count for the unknown parameters.

This study introduces a correction function $g$ to compensate for the systematic error in the measurements, as shown in Eq. (5). The correction function of one sensor is formulated with offsetting constants and its measurement $M$. The specific equation is based on a characteristic of the systematic sensor error. A single sensor can have different correction functions according to the different error characteristics over the working conditions. When the systematic errors are identical in a specific measurement range (working stage), the measurements in this range can be calibrated from one correction function. For the relative measurements in Eq. (4), working sensors also have correction functions, as in Eq. (6). Once the relative measurements are substituted with the corresponding correction functions in the benchmark as in Eq. (4), the benchmark can be finally formulated by Eq. (7). Fig. 1 shows the difference between the suggested EVIC method and the previous method [16] in terms of the benchmark formulation.

$$
\begin{aligned}
& Y_{c}=g\left(M, x_{1}, x_{2}, \ldots, x_{k}\right) \\
& Y_{c, v r}=g\left(M_{v r}, x_{v r 1}, x_{v r 2}, \ldots, x_{v r k}\right) \\
& Y_{b}=f\left(Y_{c, v 1}, Y_{c, v 2}, \ldots, Y_{c, v r}, x_{u 1}, x_{u 2}, \ldots, x_{u q}\right)
\end{aligned}
$$


where, $Y_{c}$ is the corrected measurement for the working sensor, $g$ is the correction function for the working sensors, $x$ is the offsetting constant, $k$ is the counter for the offsetting constants, and $Y_{c, v r}$ is the corrected measurement of the $r$ th relative sensor.

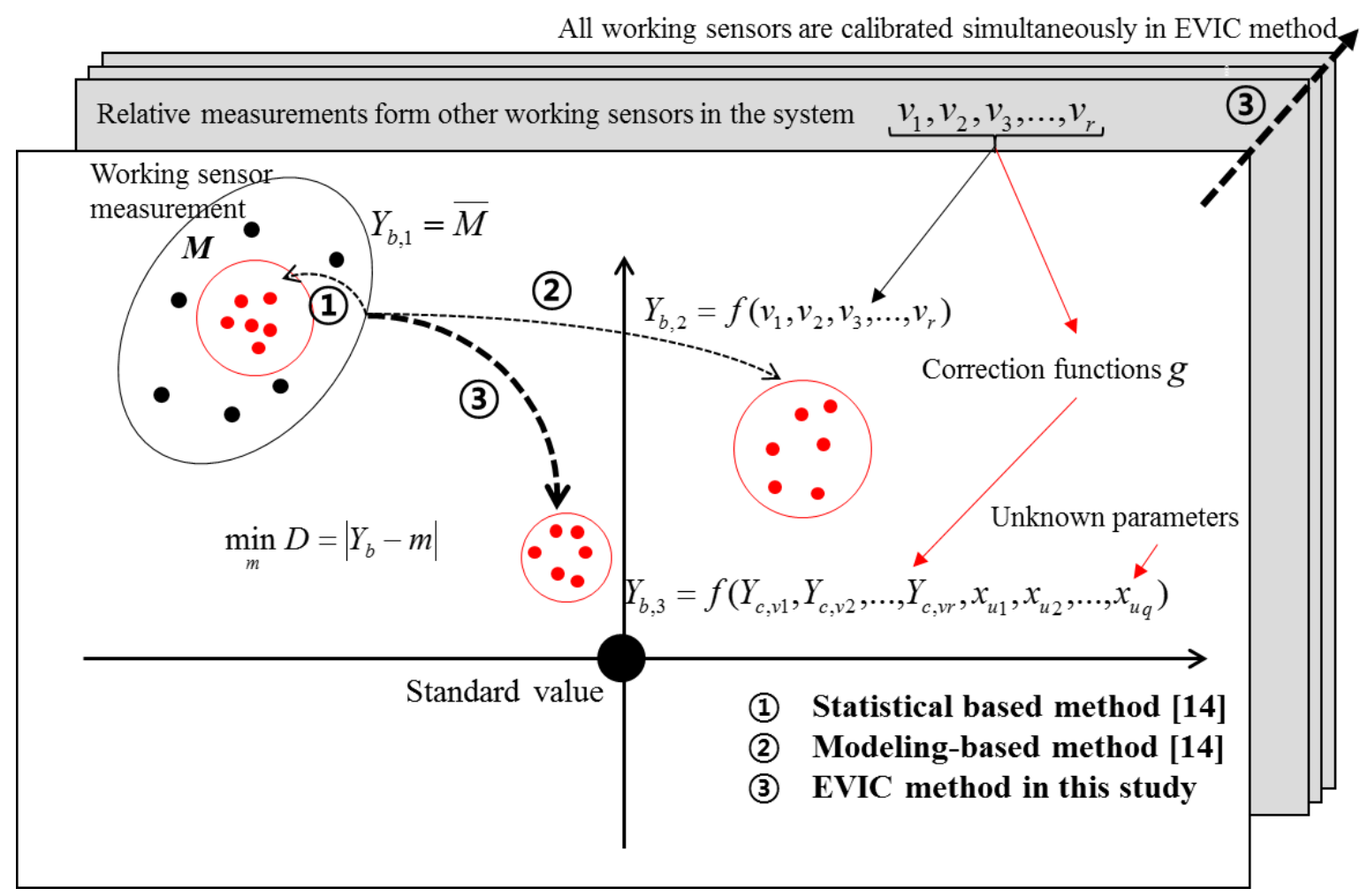

Fig. 1. A comparison of three sensor calibration methods regarding benchmark

\subsection{Distance function between benchmark and correction function}

This study suggests a distance function of EVIC from the Residual Sum of Squares (RSS) to derive variables of interest that will minimize the difference between the benchmark values and corrected values from the correction function for every working sensor in a system, as shown in Eq. (8).

$$
D(x)=\sum_{i=1}^{N}\left(Y_{b i}-Y_{c i}\right)^{2}
$$

where, $D$ is the distance function that denotes the sum of squared differences between the benchmark values and corrected values for every working sensor, $i$ is the counter for each working sensor, $x$ is the variables of EVIC problem, and $N$ is the number of benchmarks in distance function.

Unlike the existing distance function as in Eq. (1), all working sensors in a specific system can be contained and their measurements are replaced with the corresponding correction functions in the proposed distance function. Accordingly, the variables of this calibration are composed of the offsetting constants of all correction functions and the unknown parameters of the benchmark functions which are modeled by the system model. Their true values are used throughout the calibration process to minimize the distance function.

When the number of variable is more than the number of equations, the distance function in Eq. (8) is under-determined. In this case, many different combinations of the variables can be derived from the calibration even though they may differ from their true values. In order to obtain accurate 
variables, the under-determined calibration problem should be constrained by decreasing the number of unknown conditions and/or increasing the number of related equations within the calibration system. This method introduces reliable output variables of a building system and multiple timestamps. When the accurate values of the output variables such as a heat transfer rate and energy consumption are known, these values are directly defined without their correction functions and are represented in the distance function of Eq. (9).

$$
D(x)=\sum_{i=1}^{T}\left[\sum_{i=1}^{N}\left(Y_{b i}-Y_{c i}\right)^{2}+\sum_{h=1}^{H}\left(Y_{b h}-Y_{R h}\right)^{2}\right]
$$

where, $Y_{R}$ is the known system output values from reliable sensors, $h$ is the counter for the reliable output variables, $H$ is the number of reliable output variables, and $T$ is the number of sets of steady-state measurements.

Using multiple sets of steady-state measurements $(T)$ from various timestamps encourages the calibration problem to be determined by adding new relative equations. The left term on the right side represents the sensor calibration. In EVIC, the model calibration and the sensor calibration are conducted simultaneously and accurate estimates of the unknown parameters help to derive the true offsetting constants and vice versa. Such a relationship allows the measurements to be more accurate for every working sensor in a building system.

\subsection{Bayesian inference}

Deterministic or statistical approaches have been applied to a calibration method in previous studies [24-26]. In consideration of the fact that measurements from working sensors and unknown parameters can be expressed by probability distributions based on the Central Limit Theorem [25,26] and previous research $[25,29,30]$, respectively, the statistical approach is more appropriate for solving the proposed calibration problem. In this calibration, Bayesian inference is used to statistically derive the offsetting constants and unknown parameters in order to minimize the distance function of the calibration problem.

According to Bayes' theorem shown in Eqs. (10) and (11), Bayesian inference derives a posterior distribution $P\left(x \mid Y_{b}\right)$ of variables, $x$, based on a likelihood function $P\left(Y_{b} \mid x\right)$ and a prior distribution, $\pi(x)$, of the variables, $x$. The likelihood function can be based on the conditional probability distribution using a Gaussian distribution with a zero-mean $[25,26,30]$. This study plugs the distance function $D(x)$ of Eq. (9) into the Gaussian distribution, as shown in Eq. (12), so that the likelihood can be maximized when the distance function is minimized. Thus, the posterior distributions may have a higher probability when the value of the distance function is smaller. The prior distribution can be defined by a probability distribution or a range with equal probability.

$$
\begin{aligned}
& P\left(x \mid Y_{b}\right)=\frac{P\left(Y_{b} \mid x\right) \times \pi(x)}{P\left(Y_{b}\right)} \\
& P\left(Y_{b}\right)=\int P\left(Y_{b} \mid x\right) \pi(x) d x \\
& P\left(Y_{b} \mid x\right)=\frac{1}{\sigma \sqrt{2 \pi}} \exp \left[-\frac{1}{2 \sigma^{2}} D(x)\right]
\end{aligned}
$$

where, $x$ is the variables, $Y_{b}$ is the benchmark, $\pi(x)$ is the prior distribution of the variables, $P\left(x \mid Y_{b}\right)$ is the posterior distribution of the variables, $P\left(Y_{b} \mid x\right)$ is the likelihood function, $P\left(Y_{b}\right)$ is the normalizing constant, and $\sigma$ is the standard deviation. 
In the Bayesian inference, it is significantly difficult to calculate the normalizing constant as in Eq. (10) using a mathematical approach. In this study, a Markov Chain Monte Carlo (MCMC) method was used to derive a posterior distribution without calculating the integration. The MCMC method is Monte Carlo integration using Markov Chains [31,32]. To run the MCMC method, this study used the Metropolis-Hastings algorithm [33,34], which is a popular sampling technique for obtaining a sequence of random samples from joint multivariate distributions. This calibration process using Bayesian inference with MCMC method was implemented in MATLAB.

\section{Methodology of solving EVIC problem}

The methodology presented provides systematic procedures for formulating the EVIC problem and solving it accurately. The necessary conditions for conducting the EVIC are addressed in this methodology. The essential steps of methodology are established as follows:

1) Step 1: Define a system model and calibration domain

2) Step 2: Establish sets of steady-state measurements

3) Step 3: Conduct a local and/or whole calibration

\subsection{Defining a system model and local domain}

It is necessary to establish a steady-state model in terms of the building system including target working sensors in order to compose benchmarks of each working sensor $\left(Y_{b i}\right)$ and define local calibration areas. The steady-state model works well in an environment good for comparisons between calculated values from benchmarks and correction functions.

In the determined model, input variables, output variables, and unknown parameters are investigated. Then working sensors for the input variables and reliable sensors for the output variables should be matched with the corresponding variables of the model. Based on understanding of the relationships between input and output variables, the entire system model can be divided into several parts. For example, if input variables $\left(M_{3}\right.$ and $\left.M_{4}\right)$ are related to two different output variables $\left(y_{1}\right.$ and $y_{2}$ ) in their equations, the two equations can be defined as one part as shown in Fig. 2. Local calibration will be conducted in each part defined here. 


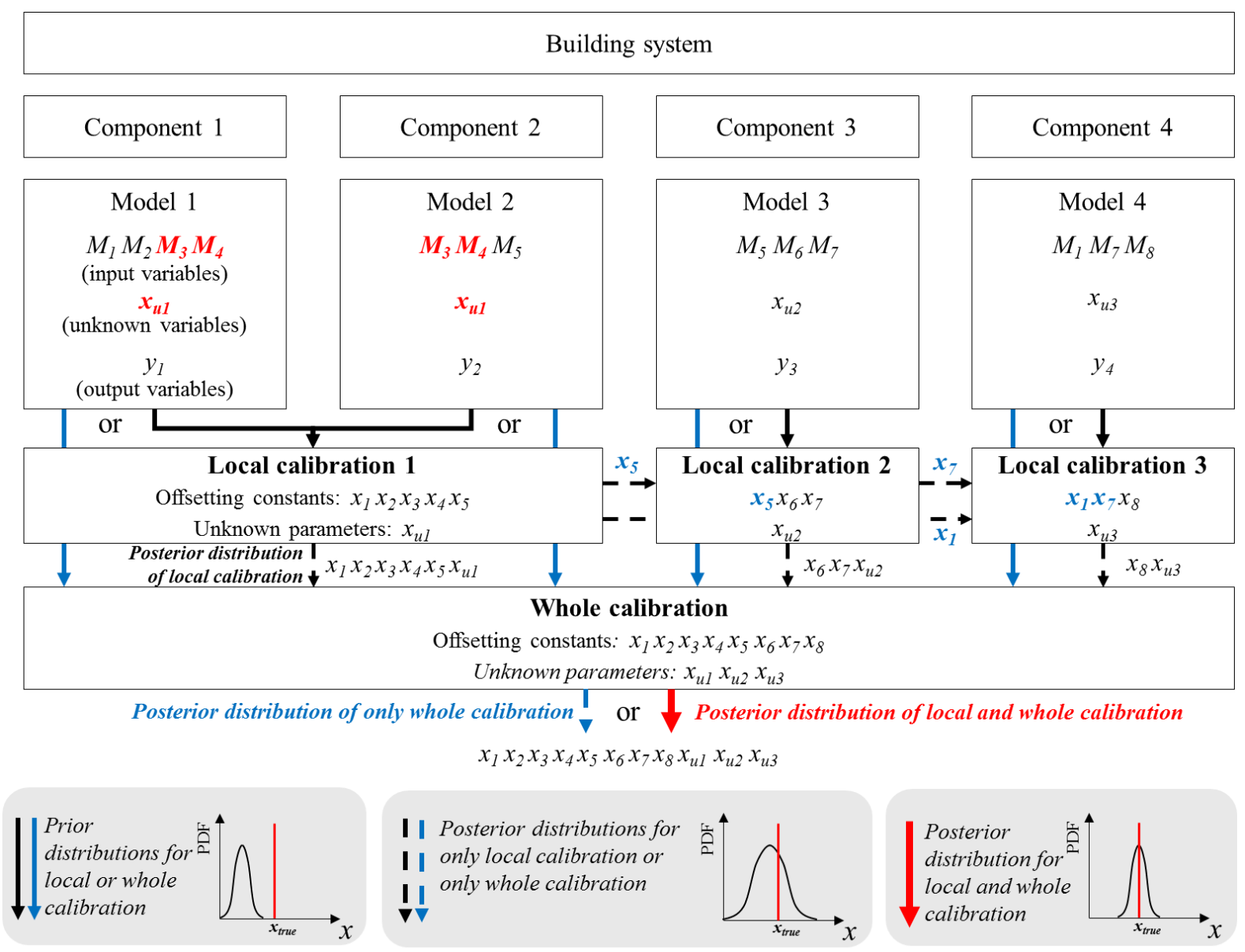

Figure 2. Diagram of defining calibration domain and local and/or whole calibration

\subsection{Establishing sets of steady-state measurements}

In order to use sets of steady-state measurements and the steady-state system model in constructing the EVIC problem having benchmarks and correction functions, the multiple values from the working sensor measured at given consecutive timestamps should be steady-state measurements. For a given duration, the steady-state measurements from $i$ th working sensor can be determined mathematically by Eq. (13).

$$
\left|m_{i, j}-\frac{\sum_{j=k}^{k+N-1} m_{i, j}}{N}\right| \leq T h_{i}
$$

where, $m$ is the measurement, $i$ is the $i$ th sensor, $j$ is the timestamp, $N$ is the number of timestamps from $k$ to $k+N-1$, and $T h$ is the defined threshold of steady condition.

When the number of readings $M_{i}=\left(m_{i, k}, m_{i, k+1}, m_{i, k+2}, \ldots, m_{i, k+N-1}\right)$ within the given time period is large enough, they are assumed to follow a normal distribution according to the Central Limit Theorem [27,28]. Then, a Student's $T$-distribution variable $(t)$ can be defined with $N-1$ degree of freedom for the $N$ samples as in Eq. (14). 


$$
t=\frac{\bar{M}_{i}-\mu}{S / \sqrt{N}}
$$

where, $t$ is the $T$-distribution variable, $\mu$ is the expected average of the population, $\bar{M}_{i}$ is the mean value of the measurements from $i$ th sensor $M_{i}, S$ is the standard deviation of the measurements $M_{i}, N$ is the number of readings $M_{i}$.

With a confidence interval of $(1-\alpha)$, the expected average of the population should be within $\left[\bar{M}_{i}-t_{\alpha / 2}\left(S / \sqrt{N}, \bar{M}_{i}+t_{\alpha / 2}(S / \sqrt{N})\right]\right.$. The stability condition threshold can be found by Eq. (15).

$$
T h_{i}=t_{\alpha / 2} \cdot \frac{S}{\sqrt{N}}
$$

where, $t_{\alpha / 2}$ is the corresponding critical value of $T$-distribution.

Through the evaluation of steady-state conditions based on Eqs. (13) - (15), steady-state measurements from the working sensors and the reliable output sensors can be expressed as vectors:

$$
\begin{gathered}
M_{i, p}=\left(m_{i, k}, m_{i, k+1}, m_{i, k+2}, \cdots, m_{i, k+N-1}\right)_{1 \times N} \\
Y_{R h, q}=\left(y_{R h, k}, y_{R h, k+1}, y_{R h, k+2}, \cdots, y_{R h, k+N-1}\right)_{1 \times N}
\end{gathered}
$$

where, $M_{i, p}$ is the vector of the $p$ th steady-state measurements from the working sensor $i, Y_{R h, q}$ is the vector of the $q$ th steady-state measurements from the reliable output sensor $h, k$ is the first timestamp in the defined steady-state period, and $N$ is the number of measurements in the defined steady-state period.

For each local calibration domain $(j)$ defined in the previous section, if all values involved in the particular calibration domain are considered as steady-state measurements at the same period of time, a set of steady-state measurements in the calibration domain is defined by Eq. (18), using the average values of their vectors from Eqs. (16) and (17). It can be easier to obtain the steady-state sets when we use the local calibration, which is applied to the sub-components, instead of a whole building system.

$$
S_{n}^{j}=\left(\bar{M}_{1}, \bar{M}_{2}, \ldots, \bar{M}_{i}, \bar{Y}_{R 1}, \bar{Y}_{R 2}, \ldots, \bar{Y}_{R h}\right)_{1 \times(I+H)}
$$

where, $S_{n}^{j}$ is the vector (a set of steady-state measurements) of the average values of relative steady-state measurements at the $j$ th local calibration domain, $n$ is the counter for the vectors $S, I$ is the number of working sensors, and $H$ is the number of reliable output sensors.

Meanwhile, each working stage of a sensor is determined considering the change of systematic sensor error within the entire working range. It is assumed that all systematic errors of different steady-states $(n)$ for the working sensor are identical in the same working stage. To assign a working stage to all steady-state measurements in the vector as in Eq. (18), their working stage is evaluated from lower and upper bounds of each working stage for the working sensor, as shown in Eqs. (19) and (20). And then, each measurement in Eq. (18) has a subscript regarding the determined working stage as in Eq. (21).

$$
\begin{gathered}
l b_{i, l} \leq \bar{M}_{i}<u b_{i, l} \\
l b_{h, l} \leq \bar{Y}_{R h}<u b_{h, l}
\end{gathered}
$$




$$
S_{n}^{j}=\left(\bar{M}_{1, l}, \bar{M}_{2, m}, \ldots, \bar{M}_{i, o}, \bar{Y}_{1, l}, \bar{Y}_{2, m}, \ldots, \bar{Y}_{R h, o}\right)_{1 \times(I+H)}
$$

where, $l b$ is the lower bound of the working stage, $u b$ is the upper bound of the working stage, and $l, m$, and $o$ are counters for the working stages.

Finally, by comparing element by element in terms of working stages of steady-state measurements for every set $\left(S_{n}^{j}\right)$ as in Eq. (21), sets of steady-state measurements having the identical elements are collected as shown in Eq. (22).

$$
S_{s e t, k}^{j}=\left(S_{1}^{j}, S_{2}^{j}, S_{3}^{j}, \ldots, S_{t}^{j}\right)_{1 \times T}
$$

where, $S_{s e t, k}^{j}$ is the vector correcting the same sets of steady-state measurements $S_{n}^{j}, k$ is the counter for the vectors $S_{\text {set }}, t$ is the counter for the sets $S_{n}$, and $T$ is the number of the sets $S_{t}$.

In the evaluation of sets of steady-state measurements, more narrow ranges in Eqs. (19) and (20) allow all bias errors to be offset approximatively by using their linear equations. However, it results in increasing sets of steady-state measurements $k$ and decreasing the number $T$ in each set $k$ because a great variety of measurement sets as in Eq. (21) should exist due to the narrow ranges. In such a circumstance, conducting a calibration for many sets $k$ in the following step could be timeconsuming and the calibration results may be less accurate with the limited number $T$. Consequently, it is important to define the working stages for each sensor reasonably. Fig. 3 suggests the conceptual procedures of establishing sets of steady-state measurements based on the defined equations in this step.

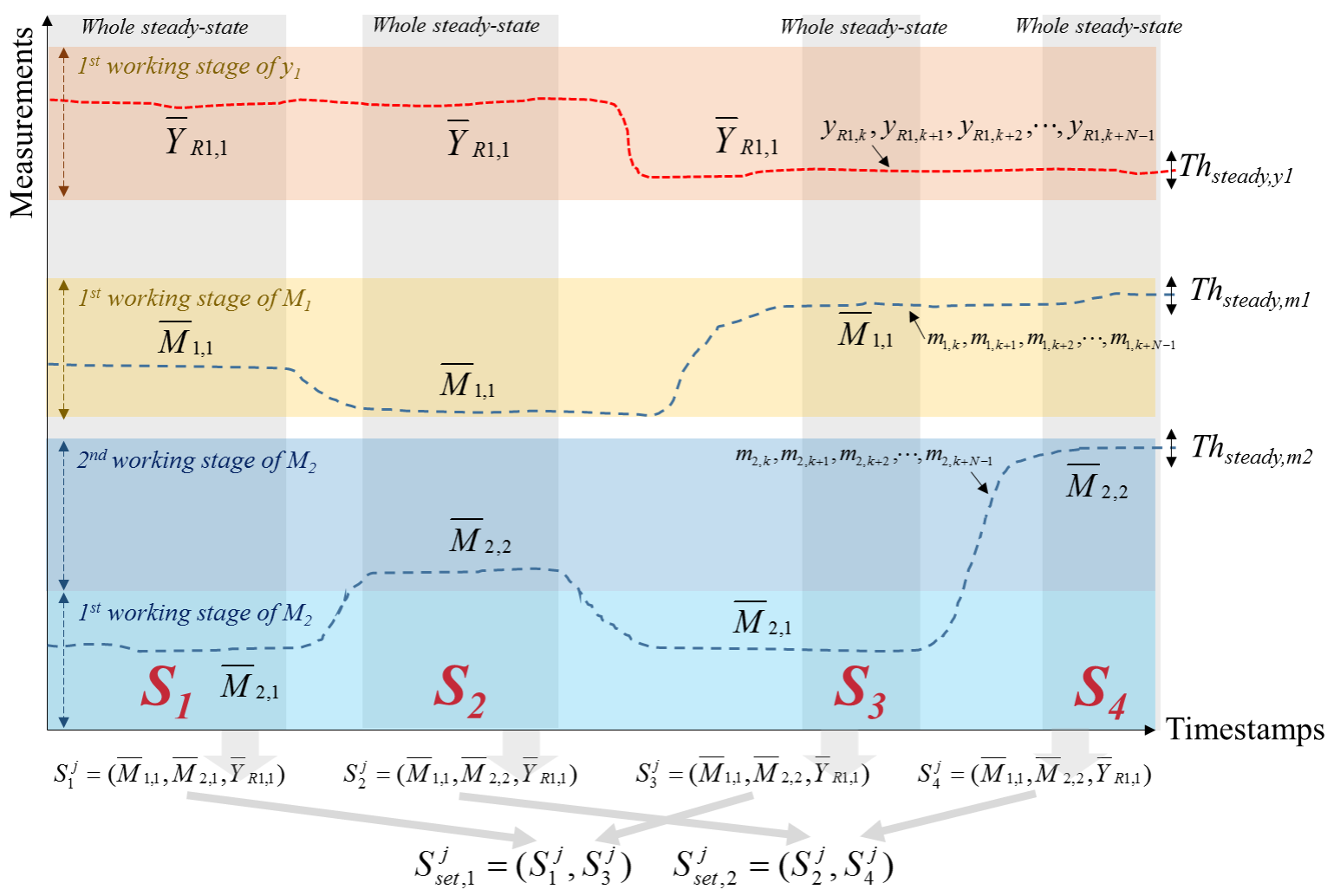

Figure 3. Illustration of establishing sets of steady-state measurements

The aforementioned EVIC methodology is further illustrated as a flowchart as in Fig. 4. 


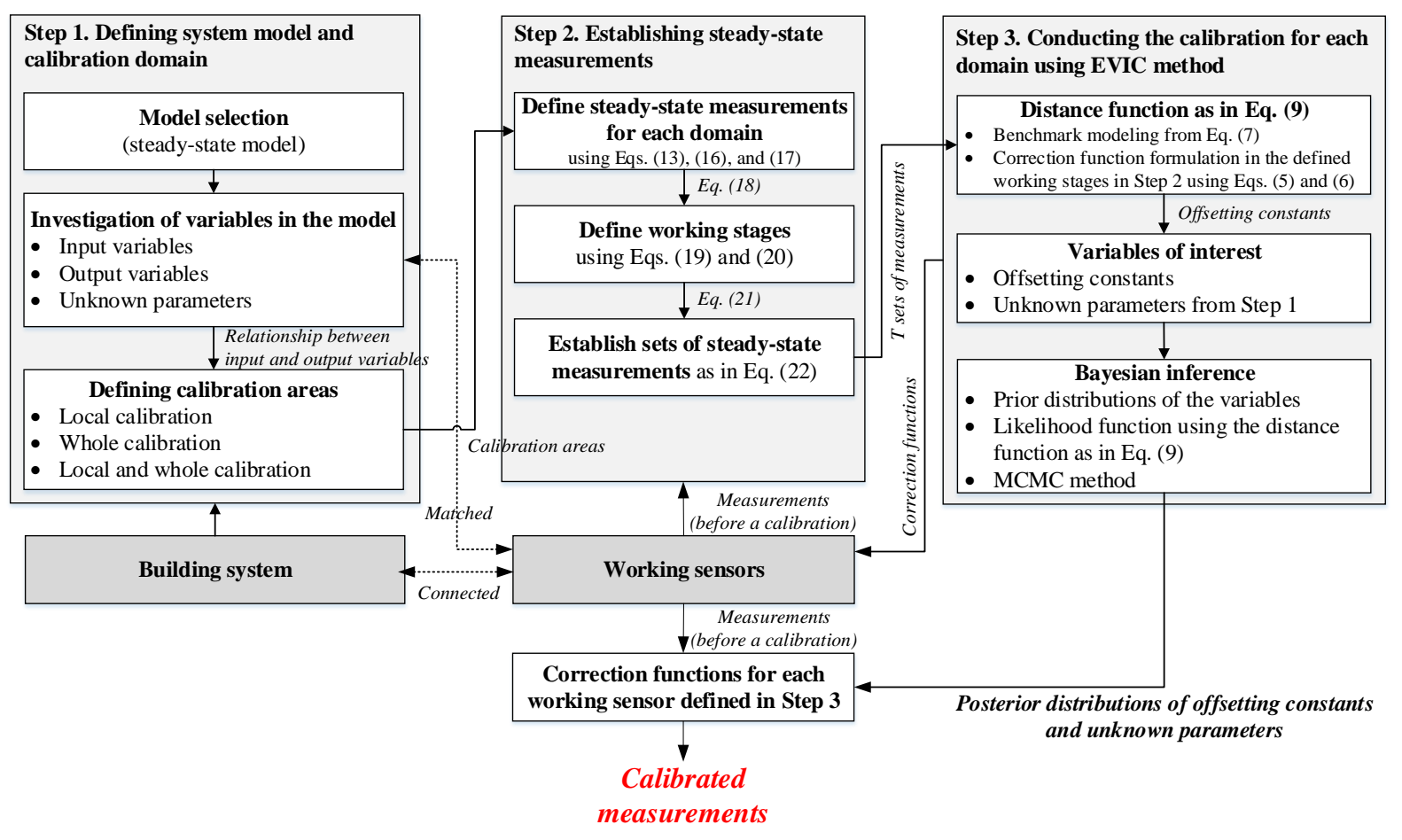

Figure 4. Methodology for the EVIC problem

\subsection{Conducting a local and/or whole calibration}

This calibration method consists of three approaches: (1) whole calibration, (2) local calibration, and (3) local and whole calibration. Fig. 2 shows each calibration approach. The whole calibration and the local calibration can calculate the posterior distributions by conducting the EVIC for a whole calibration area and each local domain, respectively. On the other hand, the local and whole calibration is conducted by a two-step process. The later whole calibration process defines the posterior distributions acquired from the former local calibration as its prior distributions, as shown in Fig. 2.

For conducting the EVIC at each local domain or the whole domain, the correction functions of each working sensor are formulated over its all working stages. The average values of the steadystate measurements in Eq. (21) are used as the measurements ( $M$ or $\left.M_{v r}\right)$ of correction functions. Then the benchmarks corresponding to working sensors and reliable output sensors are defined, respectively, from the determined system model, including the unknown parameters in Step 1 and the relative correction functions. When formulating the benchmarks at each local calibration, it is impossible to directly define some properties such as density and specific heat when they are determined based on measurements involved in other local calibration areas. For the calculation of the property, this study thus uses a mean value of each working stage in which the relative measurements are included and then the property is recalculated by the calibrated measurement during the whole calibration (if the local and whole calibration approach is selected). Finally, the distance function is constructed using the benchmarks, correction functions, and reliable output values from each local calibration or the whole calibration, as in Eq. (9). The $T$ sets of steady-state measurements, as in Eq. 22, are employed in this distance function. This is plugged into the Bayesian inference likelihood function to statistically derive the true values.

Variables of interest in this calibration problem are defined by the constants of correction functions and the unknown parameters of the system model in order to maximize the likelihood 
function by minimizing the distance function. For the offsetting constants, the prior distributions are determined according to the measured values from the corresponding working sensor in a steady-state condition. The prior distribution of unknown parameters can be determined based on previous literature and domain knowledge for a target system.

Based on the defined distance function and the prior distributions of variables in each calibration domain, a calibration is conducted for all sets $k$, as in Eq. (22), to derive the posterior distributions. The measurements from working sensors can be calibrated using the calculated posterior distribution regarding their offsetting constants and the correction functions, as shown in Fig. 2.

\section{Case study}

A case study was carried out to verify whether the suggested method can formulate the calibration problem and solve it properly before applying it to real-world applications. In this case study, various sensor errors were assigned and the calibrated results from the EVIC were compared to the pre-defined true values for working sensors.

\subsection{EVIC problem formulation for ARS}

This case study used a single-effect $\mathrm{LiBr}-\mathrm{H}_{2} \mathrm{O}$ absorption refrigeration system (ARS) to apply and demonstrate the suggested sensor calibration method. The system consisted of an absorber, condenser, generator, evaporator, solution heat exchanger, refrigerant expansion valve, solution expansion valve, and solution pump. Sensors for temperature, pressure, mass flow rate, concentration of solution, etc., were used in the ARS to provide information on how the system performances. As other building systems, the ARS is a closed system, difficult to measure accurately, and with limited number of sensors (one or none for each phenomenon). Fig. 5 shows a configuration and a flow diagram of the ARS.

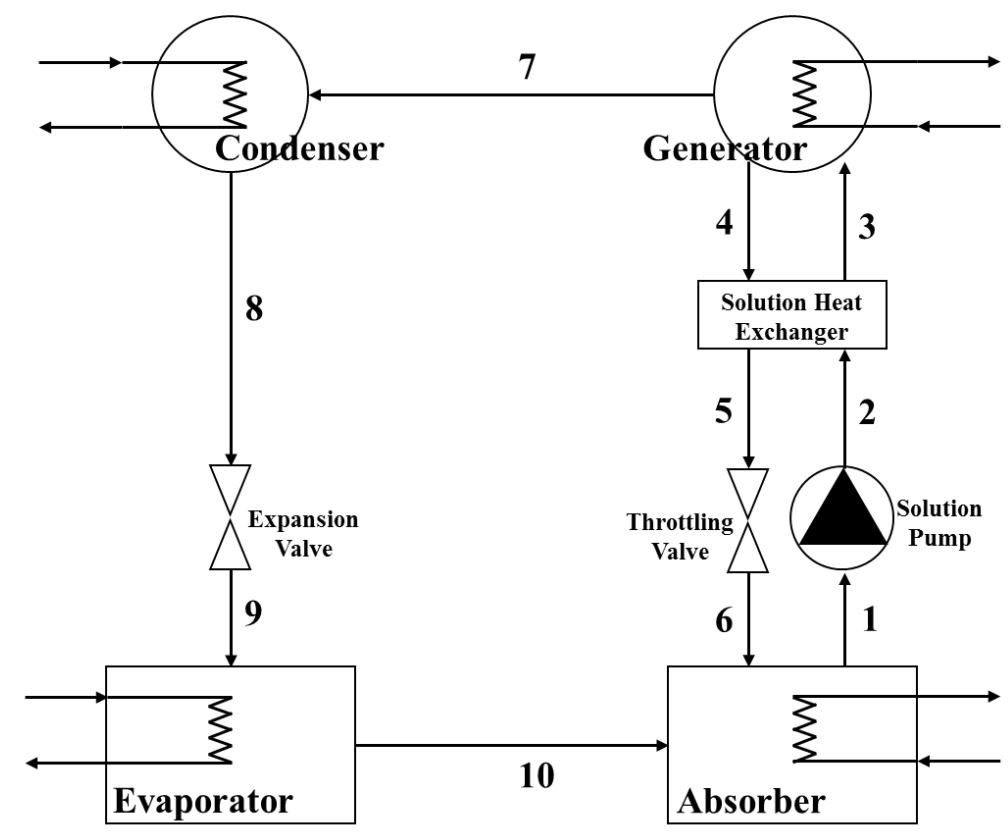

Figure 5. Flow diagram of the single-effect $\mathrm{LiBr}-\mathrm{H} 2 \mathrm{O}$ refrigeration system

The steady-state thermodynamic model was established in MATLAB (ver. 2015b), as shown in Table 1, from previous studies [35-37], which was developed based on the governing equations of the mass and energy conservation in the ARS. This model has the following calculation process: (1) solution concentrations, (2) temperatures of states 5 and 3, (3) enthalpies except for state 3, (4) mass flow rates of solutions and refrigerants, (5) enthalpy of state 3, (6) heat transfer rate at each 
component, and (7) pump work.

Table 2 shows the heat transfer rate at each component and COP through the developed model with input data in order to verify this model. The input data were defined in previous studies $[38,39]$ and those studies also show similar results using their thermodynamic model and these input data. Table 3 shows a comparison between our results and their results. From the deviation values in Table 3, it is clear that the model results are considerably similar to the results of Rubio-Maya [38]. The results of Ebrahimi, et al. [39], also show a good agreement with our results with deviations less than $5.5 \%$. These small deviations are due to equation differences in calculating thermodynamic properties of each model of the ARS.

Table 1. Reference models of the $\mathrm{LiBr}-\mathrm{H}_{2} \mathrm{O}$ ARS

\begin{tabular}{|c|c|}
\hline Building system & A single-effect $\mathrm{LiBr}-\mathrm{H}_{2} \mathrm{O}$ AR system \\
\hline Steady-state model & $\begin{array}{l}\text { Pressures of the system: Sun [35] } \\
\text { Refrigerant (water) properties: Lansing [36] } \\
\text { Absorbent (LiBr) concentration: Kaita [37] } \\
\text { Solution (LiBr-water) properties: Kaita [37] }\end{array}$ \\
\hline Input & $\begin{array}{l}\text { Absorber temperature, } T_{1} \\
\text { Generator temperature, } T_{7} \\
\text { Condenser temperature, } T_{8} \\
\text { Evaporator temperature, } T_{10} \\
\text { Solution heat exchanger effectiveness, } \varepsilon \\
\text { Evaporator cooling load, } Q_{e}{ }^{{ }^{1}, m}\end{array}$ \\
\hline Output & 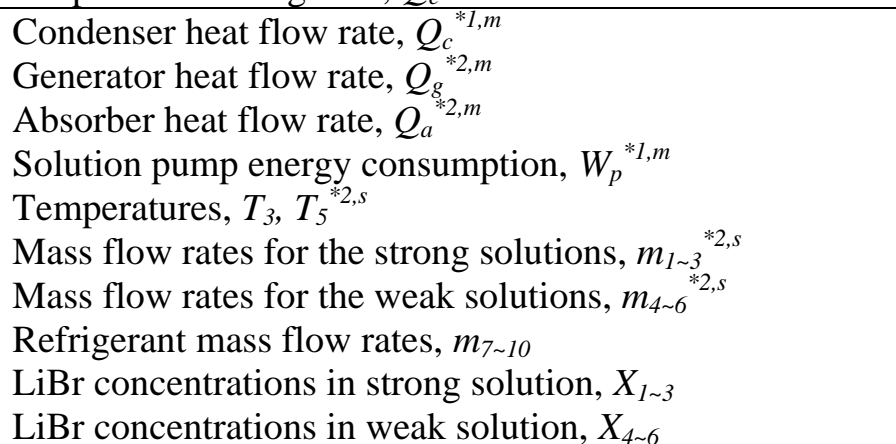 \\
\hline
\end{tabular}

1: $1^{\text {st }}$ local calibration area, $2: 2^{\text {nd }}$ local calibration area, $m$ : benchmark in the model calibration term, and $s$ : benchmark in the sensor calibration term

Table 2. Input parameter of a $\mathrm{LiBr}-\mathrm{H}_{2} \mathrm{O}$ absorption refrigeration model

\begin{tabular}{|c|c|c|}
\hline Input parameter & Symbol (unit) & Value from Rubio-Maya et al. [38] \\
\hline Evaporator cooling load & $Q_{e}(\mathrm{~kW})$ & 201.29 \\
\hline Generator temperature & $T_{7}\left({ }^{\circ} \mathrm{C}\right)$ & 84.8 \\
\hline Condenser temperature & $T_{8}\left({ }^{\circ} \mathrm{C}\right)$ & 39.8 \\
\hline Evaporator temperature & $T_{10}\left({ }^{\circ} \mathrm{C}\right)$ & 8.6 \\
\hline Absorber temperature & $T_{1}\left({ }^{\circ} \mathrm{C}\right)$ & 35.5 \\
\hline Solution heat exchanger effectiveness & $\varepsilon(\%)$ & 70.7 \\
\hline
\end{tabular}

Table 3. Verification of the suggested model through the comparison of our results with those of reference models

\begin{tabular}{|c|c|c|c|}
\hline Output parameter & $\begin{array}{c}\text { Present study } \\
(\text { deviations, \%) }\end{array}$ & $\begin{array}{c}\text { Ebrahimi et al. } \\
{[39]}\end{array}$ & $\begin{array}{c}\text { Rubio-Maya et al. } \\
{[38]}\end{array}$ \\
\hline Generator heat flow rate $(\mathrm{kW})$ & $259.685\left(5.47^{\mathrm{a}} / 0.05^{\mathrm{b}}\right)$ & 246.207 & 259.55 \\
\hline Condenser heat flow rate $(\mathrm{kW})$ & $213.373(3.50 / 0.00)$ & 206.155 & 213.37 \\
\hline
\end{tabular}




\begin{tabular}{|c|c|c|c|} 
Absorber heat flow rate $(\mathrm{kW})$ & $247.602(2.59 / 0.05)$ & 241.345 & 247.47 \\
\hline Coefficient of Performance (-) & $0.775(5.18 / 0.05)$ & 0.8175 & 0.7755 \\
\hline
\end{tabular}

a: deviation between this paper results and those of Ebrahimi et al [39]

b: deviation between this paper results and those of Rubio-Maya et al [38]

The proposed calibration approaches (local calibration only, whole calibration only, and combined calibration) were applied to working sensors for temperatures and mass flow rates at the inner ARS (states 1-10). In order to compare the calibrated results with their true values for these sensors, a virtual environment was developed using the thermodynamic model. This virtual environment can establish random sets of measurements with and without various sensor errors in each working stage of the working sensors. The results without the sensor errors can be considered as the true measurements from the working sensors. In one working stage for each working sensor, various combinations of systematic and random errors were considered. These are shown in Table 4. The deviations in Table 4 represent the systematic error based on true measurements. The systematic and random errors for mass flow rates were defined in accordance with their different scales.

Table 4. Description of error type

\begin{tabular}{|c|c|c|c|}
\hline \multirow{2}{*}{ Working sensor } & \multicolumn{2}{|c|}{ Systematic error } & $\begin{array}{c}\text { Random error } \\
\text { (standard deviation) }\end{array}$ \\
\cline { 2 - 4 } & Value (level) & Deviation & Value (level) \\
\hline$T_{1}$ & $-5^{\circ} \mathrm{C}($ Large) & $15.37 \%$ & 0.7 (Normal) \\
\hline$T_{3}$ & - & - & 1.0 (Large) \\
\hline$T_{8}$ & $-3^{\circ} \mathrm{C}$ (Normal) & $6.81 \%$ & 1.0 (Large) \\
\hline $\begin{array}{c}\text { Remaining } \\
\text { temperatures }\end{array}$ & - & - & 0.7 (Normal) \\
\hline$m_{7 \sim 10}$ & $\begin{array}{c}0.0075 \mathrm{~kg} / \mathrm{s} \\
(\text { Normal) }\end{array}$ & $8.64 \%$ & 0.0025 (Small) $^{* 1}$ \\
\hline$m_{1 \sim 6}$ & - & - & 0.035 (Normal) $^{* 2}$ \\
\hline
\end{tabular}

*1: It can be considered 0.5 in the same scale with temperatures

*2: It can be considered 0.7 in the same scale with temperatures

Five cases were designed in this study for a comparison: whole calibration (Case 1), local calibration (Case 2), local and whole calibration (Case 3), temperature calibration (Case 4), and model calibration (Case 5). Cases 1, 2, and 3 were based on the suggested EVIC method. Case 4 is related to the previous VIC method proposed in [16]. In Case 4, it was assumed that mass flow rates which are relative variables of the corresponding temperatures were accurate. In Case 5, the only way the solution's heat exchanger effectiveness $(\varepsilon)$ can be calibrated is under the assumption that all working sensors are reliable. This case can be considered a typical model calibration that estimates the accurate unknown parameters of the model by comparing the measured data to the calculated results from the model.

The entire system was divided into two parts for a local calibration based on the relationships between input and output variables in the system model. The first local calibration includes the evaporator, condenser, and solution pump. The second local calibration is conducted for the absorber and generator.

At each local calibration area, and the whole area, each threshold for working sensors was determined, as listed in Table 5. This study selected the relatively large threshold of temperature measurements to include the large random error in this calibration problem. In addition to that, this case study handles one working stage for each working sensor based on the lower and upper bounds as shown in Table 5. Based on the selected thresholds, steady-state measurements were established within the working stage. For every case study, four sets of steady-state measurements, as in Eq. (22), were collected. 
Table 5. The working stage and the steady-state threshold for each working sensor

\begin{tabular}{|c|c|c|c|c|}
\hline & $\begin{array}{c}\text { Temperatures } \\
\left(T_{1 \sim 10}\right) \\
{\left[{ }^{\circ} \mathrm{C}\right]}\end{array}$ & $\begin{array}{c}\text { Refrigerant mass } \\
\text { flow rates }\left(m_{7 \sim 10}\right) \\
{[\mathrm{kg} / \mathrm{s}]}\end{array}$ & $\begin{array}{c}\text { Mass flow rates for } \\
\text { the strong solutions } \\
\left(m_{1 \sim 3}\right)[\mathrm{kg} / \mathrm{s}]\end{array}$ & $\begin{array}{c}\text { Mass flow rates for } \\
\text { the weak solutions } \\
\left(m_{4 \sim 6}\right)[\mathrm{kg} / \mathrm{s}]\end{array}$ \\
\hline Thresholds & \pm 3.0 & \pm 0.005 & \pm 0.1 & \pm 0.1 \\
\hline Lower bounds & $+1.25^{*}$ & 0.0862 & 0.68 & 0.59 \\
\hline Upper bounds & $-1.25^{*}$ & 0.0870 & 0.88 & 0.79 \\
\hline
\end{tabular}

*: The lower and upper bounds for each temperature are from the average values of working stages

The distance function for each case, as in Eq. (9), was comprised of the benchmarks and correction functions. The correction functions were determined as linear equations for all working sensors which are calibrated in each case, as shown in Table 6. For normalization, known coefficients ( $a$ and $b$ ) were introduced into the correction functions of mass flow rates in order to match their prior distributions to those of temperatures. At the first local calibration area of Case 2, using the relative correction functions, the benchmarks were formulated for the reliable output variables (condenser heat flow rate, evaporator cooling load, and pump energy consumption) in the model calibration term of the distance function. At the second local domain of Case 2, the benchmarks of the reliable output variables (generator heat flow rate and absorber heat flow rate) were defined in the model calibration term. The benchmarks for the temperatures $\left(T_{3}\right.$ and $\left.T_{5}\right)$ and the mass flow rates $\left(m_{1 \sim 3}\right.$ and $\left.m_{4 \sim 6}\right)$ were involved in the sensor calibration term at second local domain. For the whole calibration of Cases 1 and 3-5, all benchmarks mentioned above were included in one distance function. There were four sets of steady-state measurements $(T)$ of all cases from the pre-collected data sets. The defined distance function of each case was inserted into the Bayesian inference likelihood function.

Variables of each case were determined according to the selected working sensors for a calibration and the unknown parameter of the ARS model. In Cases 1-3, offsetting constants for all temperature and mass flow rate sensors and the solution heat exchanger effectiveness $(\varepsilon)$ were defined as the variables. In Case 4, The variables were defined by the offsetting constants of every temperature sensor without the unknown parameter, $\varepsilon$. In Case 5, the solution heat exchanger effectiveness $(\varepsilon)$ was selected as the variable. In every case, the prior distributions of the defined variables were assumed to obey a normal distribution according to the Central Limit Theorem $[27,28]$. The means of every prior distribution for offsetting constants were set at 0 because the measurements taken before a calibration are considered "accurate" values. The standard deviations followed those of the measurements, as shown in Table 4. In Case 3, the prior distributions were derived by the posterior distributions obtained from each local calibration (Case 2). Every mean and standard deviation of variables in each case was listed in Tables 6 and 7.

\subsection{Results and discussion}

The posterior distributions in five cases were calculated by the Bayesian MCMC method and their means were compared to the true values, including the offsetting constants and the solution heat exchanger effectiveness $(\varepsilon)$, as shown in Tables 6 and 7. Based on the means of the posterior distributions and the correction functions, measurements from the working sensors were calibrated in each case. These results were compared to the true measurements and Table 8 suggested the results regarding one set among four sets in the defined working stages. The prior distributions, posterior distributions, and true values of all variables were compared in Figs. 5 and 6.

For the suggested EVIC method (Cases 1-3), the posterior distributions of Case 3 were closest to the true values, especially for erroneous sensors, followed by the results of Case 2 . The posterior distributions of Case 1 showed significant differences from the true values. This is because using the sets of steady-state measurements alone was insufficient to obtain reliable posterior distributions of the entire calibration domain at one time in a circumstance where the prior distribution 
of Case 1 did not represent the actual system properly.

When comparing the results of Case 2 and Case 3, the calibrated measurements from Case 3 were more similar to the true measurements, though Case 2 also showed reasonably similar results. In Case 2, the first local calibration assumed that the value of $T_{1}$, which was used to calculate the density in the benchmark of pump energy consumption, may be an average value of the working stage range because this value can be estimated through the second local calibration. The whole calibration after the local calibration of Case 2 can be used to reduce the difference between true and calibrated variables, caused from such an assumption of each local calibration, by conducting every local calibration simultaneously. However, the whole calibration has difficulties in solving the EVIC problem. it requires more accurate prior distributions or more sets of steady-state measurements to solve adequately the under-determined EVIC system; if these conditions are not enough, the posterior distributions would be inaccurate as shown in Case 1. Each local calibration prior to the whole calibration can be used to reasonably determine the prior distributions of the whole calibration.

In Cases 3 and 4, for the systematic errors, the calibrated results of every sensor along with the solution heat exchanger effectiveness $(\varepsilon)$ in Case 3 were closest to the true values. The maximum deviation between the calibrated and true values was $0.92 \%$. It was considered that the normal and large offset errors were corrected properly in Case 3. In Case 4, the calibrated temperature values of erroneous sensors $\left(T_{l}\right.$ and $\left.T_{8}\right)$ were also similar with the true values. However, the normal sensors had a considerable difference between the calibrated and true values because the mass flow rate sensors and the heat exchanger effectiveness were not calibrated in Case 4. That is, the existing virtual in-situ calibration method such as Case 4 had the limitation in calibrating all temperature and mass flow rate sensors in the ARS. In terms of the standard deviations of posterior distributions, almost all results of Cases 1-3 were lower than those of prior distributions. This suggests that many measurements from sensors having random errors can be more closely matched to their true values by decreasing the random errors through the suggested calibration.

The solution heat exchanger effectiveness $(\varepsilon)$ from Case 3 was most similar to the true value. Case 5 showed a substantial discrepancy $(10.41 \%)$ between the calculated result and true value. These results suggest the importance of a sensor calibration in a model calibration using the parameter estimation; if the model calibration is based on the measured values from erroneous sensors, the estimated values of unknown parameters may not be useful, such as in Case 5. The proposed calibration method in this study can estimate unknown parameters in a model of building systems while calibrating the measured values from working sensors.

Table 6. A comparative results of the local, whole, and combined calibration

\begin{tabular}{|c|c|c|c|c|c|c|c|c|c|c|}
\hline \multirow{3}{*}{ Sensor } & \multirow{3}{*}{$\begin{array}{l}\text { Correction } \\
\text { function and } \\
\text { variable } x\end{array}$} & \multirow{3}{*}{$\begin{array}{c}\begin{array}{c}\text { Virtual } \\
\text { condition }^{* 3}\end{array} \\
\begin{array}{c}\text { Systematic } \\
\text { error }\end{array}\end{array}$} & \multicolumn{2}{|c|}{$\begin{array}{l}\text { Before a calibration } \\
\text { (Prior distribution) }^{* 3}\end{array}$} & \multicolumn{6}{|c|}{$\begin{array}{c}\text { After a calibration } \\
\text { (Posterior distribution) }{ }^{* 3}\end{array}$} \\
\hline & & & \multirow{2}{*}{ Mean } & \multirow{2}{*}{$\begin{array}{c}\text { SD } \\
\text { (Random } \\
\text { error) }\end{array}$} & \multicolumn{2}{|c|}{ Case 1} & \multicolumn{2}{|c|}{ Case 2} & \multicolumn{2}{|c|}{ Case 3} \\
\hline & & & & & Mean & SD & Mean & SD & Mean & SD \\
\hline$T_{1}{ }^{*}\left(=T_{2}\right)$ & $T_{c, l}=T_{1}+x_{1}$ & -5.00 & 0.00 & 0.70 & -2.68 & 0.50 & $-4.79^{\mathrm{L} 2}$ & $0.17^{\mathrm{L} 2}$ & -4.88 & 0.50 \\
\hline$T_{3}$ & $T_{c, 3}=T_{3}+x_{3}$ & 0.00 & 0.00 & 1.00 & 0.16 & 0.98 & $0.10^{\mathrm{L} 2}$ & $0.85^{\mathrm{L} 2}$ & 0.07 & 0.37 \\
\hline$T_{4}\left(=T_{7}\right)$ & $T_{c, 4}=T_{4}+x_{4}$ & 0.00 & 0.00 & 0.70 & 0.87 & 0.55 & $-0.02^{\mathrm{L} 1}$ & $0.73^{\mathrm{L} 1}$ & 0.02 & 0.38 \\
\hline$T_{5}\left(=T_{6}\right)$ & $T_{c, 5}=T_{5}+x_{5}$ & 0.00 & 0.00 & 0.70 & 0.64 & 0.74 & $-0.25^{\mathrm{L} 2}$ & $0.62^{\mathrm{L} 2}$ & -0.21 & 0.38 \\
\hline$T_{8}{ }^{*}\left(=T_{9}\right)$ & $T_{c, 8}=T_{8}+x_{8}$ & -3.00 & 0.00 & 1.00 & -2.79 & 0.26 & $-2.98^{\mathrm{L1}}$ & $0.27^{\mathrm{L} 1}$ & -3.01 & 0.36 \\
\hline$T_{10}$ & $T_{c, 10}=T_{10}+x_{10}$ & 0.00 & 0.00 & 0.70 & 1.51 & 0.39 & $-0.06^{\mathrm{L1}}$ & $0.47^{\mathrm{L} 1}$ & 0.04 & 0.34 \\
\hline$m_{\mathrm{r}}^{*}\left(=m_{7 \sim 10}\right)$ & $m_{c, r}=m_{r}+a x_{m r}$ & 1.50 & 0.00 & 0.50 & 1.49 & 0.05 & $1.47^{\mathrm{L} 1}$ & $0.07^{\mathrm{L} 1}$ & 1.48 & 0.13 \\
\hline$m_{1}\left(=m_{1 \sim 3}\right)$ & $m_{c, l}=m_{1}+b x_{m l}$ & 0.00 & 0.00 & 0.70 & 0.11 & 0.28 & $-0.03^{\mathrm{L1}}$ & $0.26^{\mathrm{L} 1}$ & 0.01 & 0.30 \\
\hline$m_{4}\left(=m_{4 \sim 6}\right)$ & $m_{c, 4}=m_{4}+b x_{m 4}$ & 0.00 & 0.00 & 0.70 & 0.39 & 0.28 & $0.20^{\mathrm{L} 2}$ & $0.16^{\mathrm{L} 2}$ & 0.12 & 0.30 \\
\hline$\varepsilon^{* 2}$ & $x_{u l}$ & 0.600 & 0.65 & 0.070 & 0.605 & 0.026 & $0.607^{\mathrm{L} 2}$ & $0.023^{\mathrm{L} 2}$ & 0.604 & 0.026 \\
\hline
\end{tabular}

*: erroneous sensors, *2: unknown parameter, *3: temperatures $\left({ }^{\circ} \mathrm{C}\right)$ and mass flow rates $(\mathrm{kg} / \mathrm{s}), \mathrm{L} 1$ : from $1^{\text {st }}$ local calibration, L2: from $2^{\text {nd }}$ local calibration, $a$ is $1 / 200$, and $b$ is $1 / 20$.

Table 7. A comparative results of calibration methods

\begin{tabular}{l|l|l|l|l} 
Sensor & Correction & Virtual & Before a calibration & After a calibration
\end{tabular}




\begin{tabular}{|c|c|c|c|c|c|c|c|c|c|c|}
\hline & \multirow{3}{*}{$\begin{array}{c}\text { function and } \\
\text { variable } x\end{array}$} & \multirow{3}{*}{$\begin{array}{c}\text { condition }^{* 3} \\
\begin{array}{c}\text { Systematic } \\
\text { error }\end{array}\end{array}$} & \multicolumn{2}{|c|}{ (Prior distribution) $^{* 3}$} & \multicolumn{6}{|c|}{ (Posterior distribution) $^{* 3}$} \\
\hline & & & \multirow[b]{2}{*}{ Mean } & \multirow{2}{*}{$\begin{array}{c}\text { SD } \\
\text { (Random } \\
\text { error) }\end{array}$} & \multicolumn{2}{|c|}{ Case 3} & \multicolumn{2}{|c|}{ Case 4} & \multicolumn{2}{|c|}{ Case 5} \\
\hline & & & & & Mean & SD & Mean & $\mathrm{SD}$ & Mean & SD \\
\hline$T_{1}{ }^{*}\left(=T_{2}\right)$ & $T_{c, l}=T_{1}+x_{1}$ & -5.00 & 0.00 & 0.70 & -4.88 & 0.50 & -3.59 & 0.32 & - & - \\
\hline$T_{3}$ & $T_{c, 3}=T_{3}+x_{3}$ & 0.00 & 0.00 & 1.00 & 0.07 & 0.37 & 1.78 & 1.11 & - & - \\
\hline$T_{4}\left(=T_{7}\right)$ & $T_{c, 4}=T_{4}+x_{4}$ & 0.00 & 0.00 & 0.70 & 0.02 & 0.38 & 0.08 & 1.84 & - & - \\
\hline$T_{5}\left(=T_{6}\right)$ & $T_{c, 5}=T_{5}+x_{5}$ & 0.00 & 0.00 & 0.70 & -0.21 & 0.38 & 2.17 & 0.79 & - & - \\
\hline$T_{8}{ }^{*}\left(=T_{9}\right)$ & $T_{c, 8}=T_{8}+x_{8}$ & -3.00 & 0.00 & 1.00 & -3.01 & 0.36 & -2.79 & 0.99 & - & - \\
\hline$T_{10}$ & $T_{c, 10}=T_{10}+x_{10}$ & 0.00 & 0.00 & 0.70 & 0.04 & 0.34 & 0.32 & 0.54 & - & - \\
\hline$m_{\mathrm{r}}^{*}\left(=m_{7 \sim 10}\right)$ & $m_{c, r}=m_{r}+a x_{m r}$ & 1.50 & 0.00 & 0.50 & 1.48 & 0.13 & - & - & - & - \\
\hline$m_{1}\left(=m_{1 \sim 3}\right)$ & $m_{c, l}=m_{1}+b x_{m l}$ & 0.00 & 0.00 & 0.70 & 0.01 & 0.30 & - & - & - & - \\
\hline$m_{4}\left(=m_{4 \sim 6}\right)$ & $m_{c, 4}=m_{4}+b x_{m 4}$ & 0.00 & 0.00 & 0.70 & 0.12 & 0.30 & - & - & - & - \\
\hline$\varepsilon^{* 2}$ & $x_{u 1}$ & 0.600 & 0.65 & 0.070 & 0.604 & 0.026 & - & - & 0.662 & 0.04 \\
\hline
\end{tabular}

*: erroneous sensors, *2: unknown parameter, *3: temperatures $\left({ }^{\circ} \mathrm{C}\right)$ and mass flow rates $(\mathrm{kg} / \mathrm{s}), a$ is $1 / 200$, and $b$ is $1 / 20$.

Table 8. Calibrated measurements of each case

\begin{tabular}{|c|c|c|c|c|c|c|c|c|c|c|c|c|}
\hline \multirow{3}{*}{ Sensor } & \multirow{3}{*}{$\begin{array}{c}\begin{array}{c}\text { Virtual } \\
\text { condition }^{* 3}\end{array} \\
\text { True }\end{array}$} & \multirow{3}{*}{$\begin{array}{c}\begin{array}{c}\text { Before a } \\
\text { calibration }^{* 3}\end{array} \\
\text { Raw }\end{array}$} & \multicolumn{10}{|c|}{ After a calibration ${ }^{* 3}$} \\
\hline & & & \multicolumn{2}{|c|}{ Case 1} & \multicolumn{2}{|c|}{ Case 2} & \multicolumn{2}{|c|}{ Case 3} & \multicolumn{2}{|c|}{ Case 4} & \multicolumn{2}{|c|}{ Case 5} \\
\hline & & & Cali. & Dev. & Cali. & Dev. & Cali. & Dev. & Cali. & Dev. & Cali. & Dev. \\
\hline$T_{1}^{*}\left(=T_{2}\right)$ & 32.53 & 37.53 & 34.85 & 7.13 & 32.74 & 0.64 & 32.65 & 0.38 & 33.94 & 4.35 & 37.53 & 15.37 \\
\hline$T_{3}$ & 57.81 & 57.81 & 57.97 & 0.28 & 57.90 & 0.17 & 57.87 & 0.12 & 59.58 & 3.07 & 57.81 & 0.00 \\
\hline$T_{4}\left(=T_{7}\right)$ & 84.84 & 84.84 & 85.71 & 1.03 & 84.81 & 0.03 & 84.85 & 0.02 & 84.91 & 0.09 & 84.84 & 0.00 \\
\hline$T_{5}\left(=T_{6}\right)$ & 53.45 & 53.45 & 54.09 & 1.19 & 53.20 & 0.47 & 53.24 & 0.40 & 55.62 & 4.07 & 53.45 & 0.00 \\
\hline$T_{8}^{*}\left(=T_{9}\right)$ & 44.05 & 47.05 & 44.26 & 0.48 & 44.07 & 0.04 & 44.04 & 0.02 & 44.26 & 0.47 & 47.05 & 6.81 \\
\hline$T_{10}$ & 9.15 & 9.15 & 10.66 & 16.48 & 9.09 & 0.64 & 9.19 & 0.46 & 9.47 & 3.47 & 9.15 & 0.00 \\
\hline$m_{\mathrm{r}}{ }^{*}\left(=m_{7 \sim 10}\right)$ & 0.0868 & 0.0793 & 0.0868 & 0.07 & 0.0867 & 0.17 & 0.0867 & 0.11 & 0.0793 & 8.64 & 0.0793 & 8.64 \\
\hline$m_{1}\left(=m_{1 \sim 3}\right)$ & 0.7644 & 0.7644 & 0.7699 & 0.72 & 0.7631 & 0.17 & 0.7647 & 0.03 & 0.7644 & 0.00 & 0.7644 & 0.00 \\
\hline$m_{4}\left(=m_{4 \sim 6}\right)$ & 0.6776 & 0.6776 & 0.6973 & 2.90 & 0.6877 & 1.49 & 0.6838 & 0.92 & 0.6776 & 0.00 & 0.6776 & 0.00 \\
\hline$\varepsilon^{22}$ & 0.600 & 0.650 & 0.605 & 0.86 & 0.607 & 1.09 & 0.604 & 0.62 & 0.65 & 8.33 & 0.662 & 10.41 \\
\hline
\end{tabular}

*: erroneous sensors, $* 2$ : unknown parameter, and $* 3$ : temperatures $\left({ }^{\circ} \mathrm{C}\right)$ and mass flow rates $(\mathrm{kg} / \mathrm{s})$, Deviation $(\%)$.
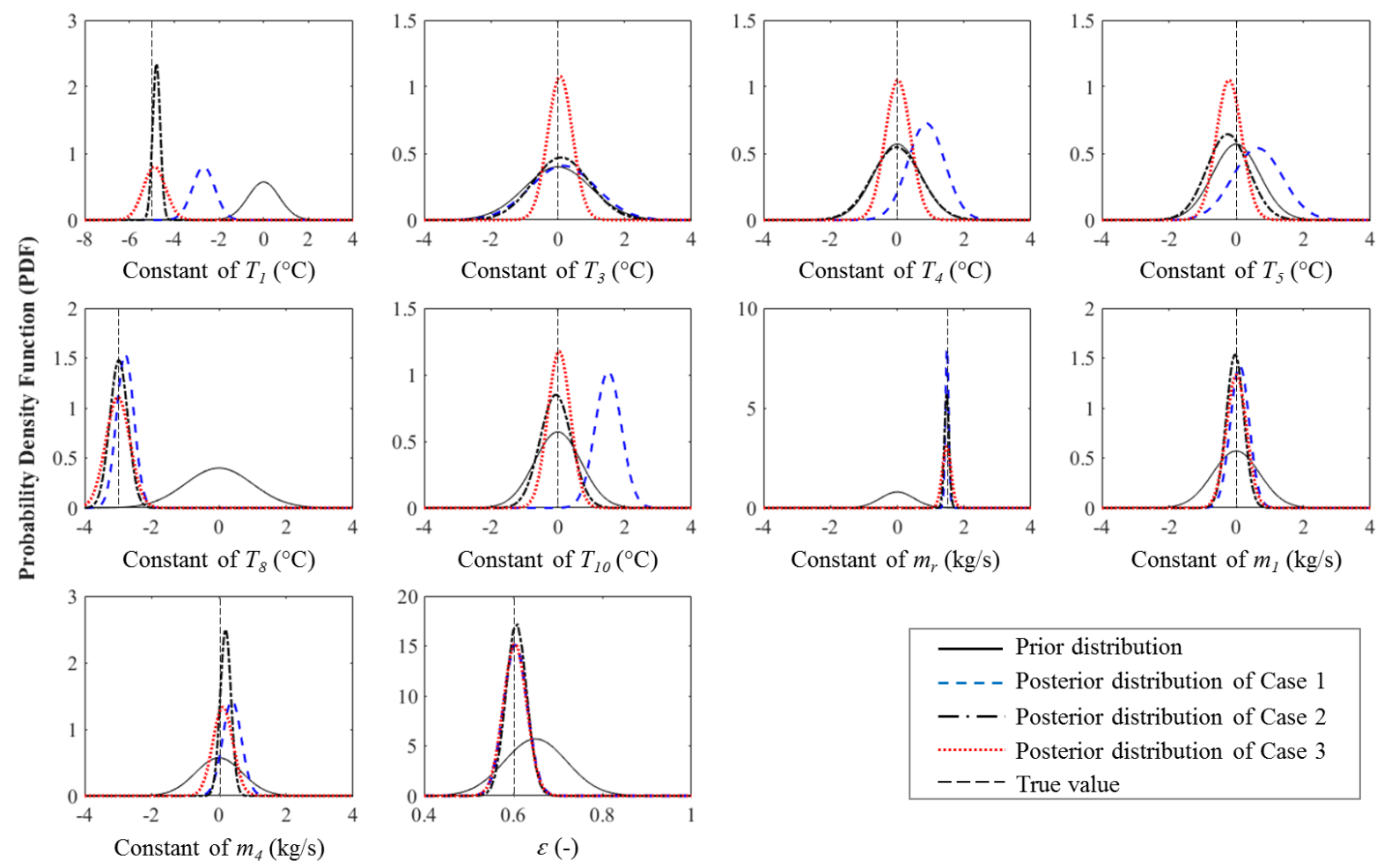

Figure 5. A comparison between prior and posterior distributions of Cases 1-3 with true values 

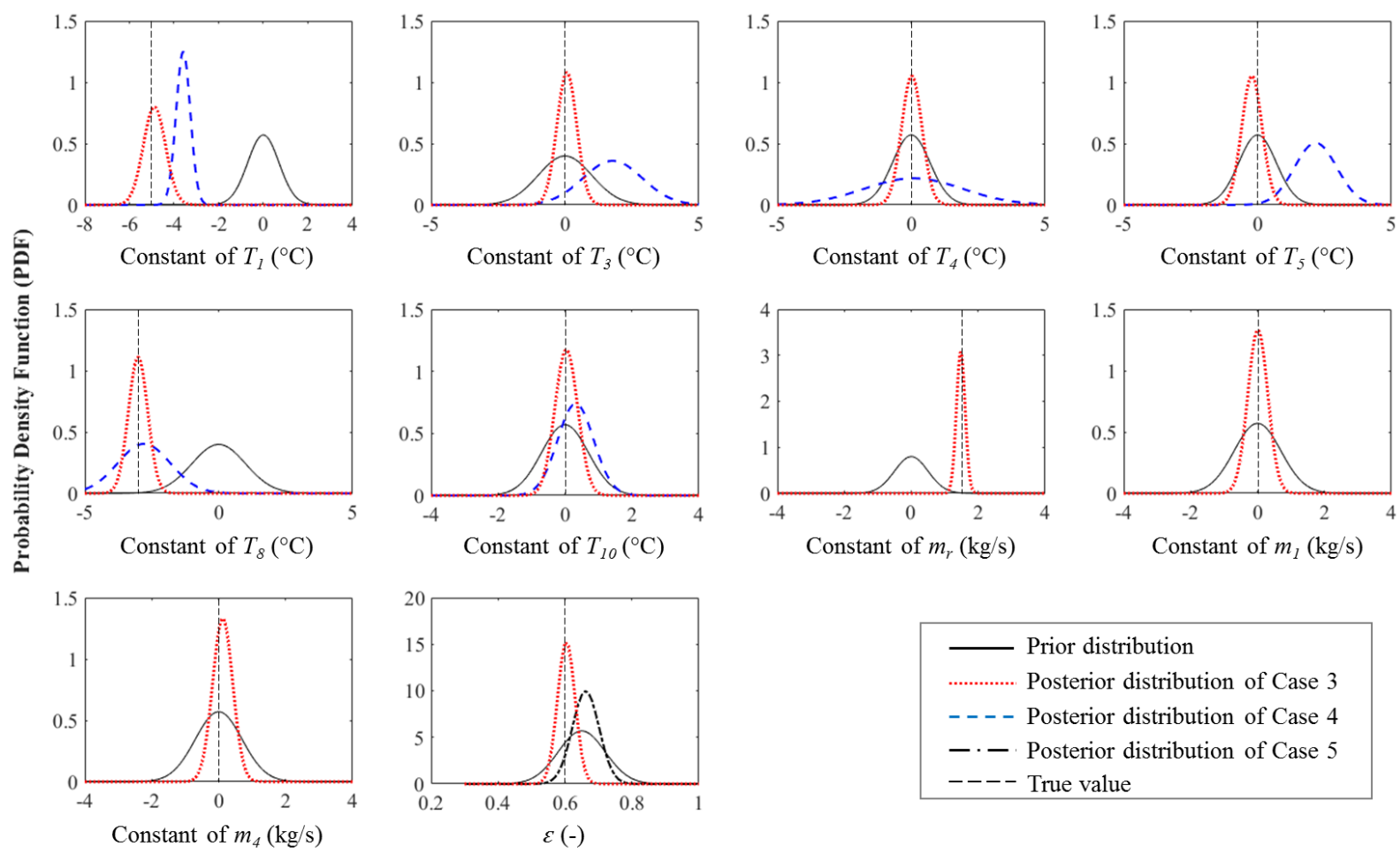

Figure 6. A comparison between prior and posterior distributions of Cases 3-5 with true values

\section{Conclusion}

In this study, the extended in-situ sensor calibration in building systems is proposed to be carried out with the EVIC problem formulation, as well as a methodology for solving the problem. This calibration problem consists of benchmarks and correction functions for all working sensors in a target system and handles both sensor calibration and model calibration. The offsetting constants of correction functions and the unknown parameters of benchmarks that are modeled by the system model are estimated statistically using Bayesian inference in order to minimize the difference between benchmarks and values of correction functions for an entire system. Through the correction functions including the estimated values of offsetting constants, the measurements from all working sensors are finally calibrated by decreasing the systematic and random errors.

The suggested methodology provides the systematic procedures to solve the EVIC problem which is inherently an under-determined system. In step 1, each local calibration is defined by understanding relationships between input and output variables in a steady-state system model. In Step 2, sets of stead-state measurements are established to add more equations to the underdetermined EVIC problem. In Step 3, based on the sets of steady-state measurements and local calibration areas, three calibration approaches are conducted. The posterior distributions of each local calibration can be proper prior distributions of a whole calibration. They could also be a major solution to solve the under-determined EVIC problem.

In this case study, the suggested method was applied to the single-effect $\mathrm{LiBr}-\mathrm{H}_{2} \mathrm{O}$ absorption refrigeration system to demonstrate the method. Unlike the existing method [16], the suggested three approaches were able to address all working sensors for temperatures and mass flow rates in the ARS. The calibrated results from a two-step approach (local and whole calibration) were closest to true measurements. The solution heat exchanger effectiveness $(\varepsilon)$, which is the unknown parameter of the ARS model, was almost the same as its true value through the model calibration together with the sensor calibration in the suggested method. The accurate unknown parameters 
enable benchmark values for working sensors to be more accurate in the sensor calibration. Meanwhile, the weaknesses of the whole calibration in defining prior distributions can be overcome by using the local calibration; if the prior distributions do not represent the systematic errors, the calibration results should be inaccurate when the calibration problem is the under-determined condition. The local calibration can establish a reasonable prior distribution.

The suggested EVIC method can be applied as a functional layer of smart building system to ensure the sensing quality and modeling reliability. In the real application, we might meet the challenges where the system is more complicated in terms of sensor number and unknown variable number. We also need to consider the reduction of computational cost and deployment of the algorithms throughout the system. Meanwhile, the sensors' systematic errors might not be static. More study is needed to handle the time or event-driven drift errors by using the proposed calibration framework.

\section{Acknowledgements}

The authors gratefully acknowledge the support of this study by the National Science Foundation under Grant No. EPS-10004094.

\section{References}

[1] Annual Energy Outlook 2013, EIA, 2013.

[2] Y. Yu, V. Loftness, D. Yu, Multi-structural fast nonlinear model-based predictive control of a hydronic heating system, Build. Environ. 69 (2013) 131-148.

[3] Y. Yu, D. Woradechjumroen, D. Yu, A review of fault detection and diagnosis methodologies on air-handling units, Energy and Buildings 82 (2014) 550-562.

[4] S. Li and J. Wen, Application of pattern matching method for detecting faults in air handling unit system, Automation in Construction 43 (2014) 49-58.

[5] H. Han, G. Gu, T. Wang, Z.R. Li, Important sensors for chiller fault detection and diagnosis (FDD) from the perspective of feature selection and machine learning, International Journal of Refrigeration 34 (2011) 586-599.

[6] S.T. Bushby, N. Castro, et al., Using the Virtual Cybernetic Building Testbed and AFDD Test Shell for AFDD Tool Development, NISTIR 6818, National Institute of Standards and Technology, Gaithersburg, MD, 2001.

[7] Y. Yao, J. Chen, Global Optimization of a Central Air-conditioning System Using Decompositioncoordination Method, Energy and Buildings 42 (5) (2010) 570-583.

[8] Y. Yao, Z. Lian, Z. Hou, X. Zhou, Optimal operation of a large cooling system based on an empirical model, Applied Thermal Engineering 24 (16) (2004) 2303-2321.

[9] V. Bychkovskiy, S. Megerian, D. Estrin, M. Potkonjak, A collaborative approach to in-place sensor calibration, Lect. Notes Comput. Sci 263 (2003) 301-316.

[10] D. Yu, H. Li, Y. Yu, J. Xiong, Virtual calibration of a supply air temperature sensor in rooftop air conditioning units, HVAC R Res. 17 (1) (2011) 31-50.

[11] V. Dulev, S. Ermishin, N. Khoteev, A. Lopatin, P. Shabanov, A. Menshikov, A. Startsev, Automatedmeasuring system designed to calibrate measuring devices using virtual standards technology, 2004 IEEE Autotestcon 2004 158-164.

[12] D. Yu, H. Li, L. Ni, Y. Yu, An improved virtual calibration of a supply air temperature sensor in 
rooftop air conditioning units, HVAC R Res. J. 17 (5) (2011) 798-812.

[13] T. Kato, Perturbation Theory for Linear Operators, Springer-Verlag, 1995, ISBN: 978-3-54058661-6.

[14] Z. Li, G. Huang, Preventive approach to determine sensor importance and maintenance requirements, Autom. Constr. 31 (2013) 307-312.

[15] H.T. Castrup, W.G. Eicke, J.L. Hayes, A. Mark, R.E. Martin, J.L. Taylor, Metrology: Calibration and measurement processes guidelines, NASA Reference Publication, June 1994.

[16] Y. Yu, H. Li, Virtual in-situ calibration method in building systems, Automation in Construction 59 (2015) 59-67.

[17] J. Fend, S. Megerian, M. Potkonjak, Model-based calibration for sensor networks, Sensors (October 2003) 737-742.

[18] L. Balzano, R. Nowak, Blind calibration of sensor networks, Proceedings of International Conference on Information Processing in Sensor Networks (IPSN) 07, April 25-27, 2007, Cambridge, Massachusetts, USA, 2007.

[19] J.W. Fisher, R.L. Moses, A.S. Willsky, Nonparametric Belief Propagation for Self-Calibration in Sensor Networks, International Conference on Information Processing in Sensor Networks (IPSN) 04, April 26-27, Berkeley, California, USA, 2004.

[20] Z. Geng, F. Yang, M. Li, N. Wu, A bootstrapping-based statistical procedure for multivariate calibration of sensor arrays, Sensors and Actuators B 188 (2013) 440-453.

[21] Z. Geng, F. Yang, X. Chen, N. Wu, Gaussian process based modeling and experimental design for sensor calibration in drifting environments, Sensors and Actuators B 216 (2015) 321-331.

[22] Z. Geng, F. Yang, N. Wu, Optimum design of sensor arrays via simulation-based multivariate calibration, Sensors and Actuators B 156 (2011) 854- 862.

[23] L. Zhang, F. Tian, C. Kadri, B. Xiao, H. Li, L. Pan, H. Zhou, On-line sensor calibration transfer among electronic nose instruments for monitoring volatile organic chemicals in indoor air quality, Sensors and Actuators B 160 (2011) 899-909.

[24] S. Yoon, J. Seo, W. Cho, D. Song, A calibration method for whole-building airflow simulation in high-rise residential buildings, Building and Environment 85 (2015) 253-262.

[25] Y. Kim, S. Yoon, C. Park, Stochastic comparison between simplified energy calculation and dynamic simulation, Energy and Buildings 64 (2013) 332-342.

[26] M. Ramin, G.B. Arhonditsis, Bayesian calibration of mathematical models: Optimization of model, Ecological Informatics 18 (2013) 107-116.

[27] ISO/IEC, Guide 98-3, Uncertainty of measurement- Part 3: guide to the expression of uncertainty in measurement, 2008.

[28] R.M. Dudley, Uniform Central Limit Theorems, Cambridge University Press, 1999, ISBN-13: 978-0521498845.

[29] I.A. Macdonald, P. Strachan, Practical application of uncertainty analysis, Energy and Buildings 33 (3) (2001) 219-227.

[30] Y.S. Heo, Bayesian calibration of building energy models for energy retrofit decision-making under uncertainty, Georgia Institute of Technology, Atlanta, GA, USA, 2011 (Ph.D. Thesis).

[31] W.R. Gilks, S. Richardson, D. Spiegelhalter, Markov Chain Monte Carlo in Practice, Chapman and Hall, 1995. 
[32] D. Gamerman, H.F. Lopes, Markov Chain Monte Carlo-Stochastic Simulation for Bayesian Inference, Chapman and Hall, 2006.

[33] N. Metropolis, A.W. Rosenbluth, M.N. Rosenbluth, A.H. Teller, E. Teller, Equations of state calculations by fast computing machines, Journal of Chemical Physics 21 (6) (1953) 1087-1092.

[34] W.K. Hastings., Monte Carlo sampling methods using Markov Chains and their applications, Biometrika 57 (1) (1970) 97-109.

[35] D.W. Sun, Thermodynamic design data and optimum design maps for absorption refrigeration systems. Appl Therm Eng 17(3) (1997) 211-221.

[36] F.L. Lansing, Computer modeling of a single-stage lithium bromide/water absorption refrigeration unit. JPL Deep Space Network Progress Report, 1976, DSN 42-32, pp. 247-257.

[37] Y. Kaita, Thermodynamic properties of lithium bromide-water solutions at high temperatures. Int J Refrig 24 (2001) 374-390.

[38] C. Rubio-Maya, J.J. Pachero-Ibarra, J.M. Belman-Flores, S.R. Galvan-Gonzalez, C. MendozaCovarrubias, NLP model of a $\mathrm{LiBr}-\mathrm{H} 2 \mathrm{O}$ absorption refrigeration system for the minimization of the annual operating cost. Appl Therm Eng 37 (2012) 10-18.

[39] K. Ebrahimi, G.F. Jones, A.S. Fleischer, Thermo-economic analysis of steady state waste heat recovery in data centers using absorption refrigeration, Applied Energy 139 (2015) 384-397. 